\title{
BMJ Open Effect of real-time visual feedback device 'Quality Cardiopulmonary Resuscitation (QCPR) Classroom' with a metronome sound on layperson CPR training in Japan: a cluster randomized control trial
}

Shota Tanaka, ${ }^{1}$ Kyoko Tsukigase, ${ }^{1}$ Takahiro Hara, ${ }^{2}$ Ryo Sagisaka, ${ }^{2}$ Helge Myklebust, ${ }^{3}$ Tonje Soraas Birkenes, ${ }^{3}$ Hiroyuki Takahashi, ${ }^{1}$ Ayana Iwata, ${ }^{1}$ Yutaro Kidokoro, ${ }^{1}$ Momoyo Yamada, ${ }^{1}$ Hiroki Ueta, ${ }^{4}$ Hiroshi Takyu, ${ }^{2}$ Hideharu Tanaka ${ }^{1,2}$

To cite: Tanaka S, Tsukigase $\mathrm{K}$, Hara T, et al. Effect of real-time visual feedback device 'Quality Cardiopulmonary Resuscitation (QCPR) Classroom' with a metronome sound on layperson CPR training in Japan: a cluster randomized control trial. BMJ Open 2019;9:e026140. doi:10.1136/ bmjopen-2018-026140

\section{- Prepublication history and} additional material for this paper are available online. To view please visit the journal (http:// dx.doi.org/10.1136/bmjopen2018-026140).

Received 25 August 2018

Revised 1 March 2019

Accepted 23 May 2019
Check for updates

(C) Author(s) (or their employer(s)) 2019. Re-use permitted under CC BY-NC. No commercial re-use. See rights and permissions. Published by BMJ.

For numbered affiliations see end of article.

Correspondence to

Mr. Shota Tanaka;

tanakamedical24@gmail.com

\section{ABSTRACT}

Objectives 'Quality Cardiopulmonary Resuscitation (QCPR) Classroom' was recently introduced to provide higher-quality Cardiopulmonary Resuscitation (CPR) training. This study aimed to examine whether novel QCPR Classroom training can lead to higher chest-compression quality than standard CPR training.

Design A cluster randomised controlled trial was conducted to compare standard CPR training (control) and QCPR Classroom (intervention).

Setting Layperson CPR training in Japan.

Participants Six hundred forty-two people aged over 15 years were recruited from among CPR trainees.

Interventions CPR performance data were registered without feedback on instrumented Little Anne prototypes for 1 min pretraining and post-training. A large classroom was used in which QCPR Classroom participants could see their CPR performance on a big screen at the front; the control group only received instructor's subjective feedback.

Primary and secondary outcome measures The primary outcomes were compression depth $(\mathrm{mm})$, rate (compressions per minute (cpm)), percentage of adequate depth (\%) and recoil (\%). Survey scores were a secondary outcome. The survey included participants' confidence regarding CPR parameters and ease of understanding instructor feedback.

Results In total, 259 and 238 people in the control and QCPR Classroom groups, respectively, were eligible for analysis. After training, the mean compression depth and rate were $56.1 \pm 9.8 \mathrm{~mm}$ and $119.2 \pm 7.3 \mathrm{cpm}$ in the control group and $59.5 \pm 7.9 \mathrm{~mm}$ and $116.8 \pm 5.5 \mathrm{cpm}$ in the QCPR Classroom group. The QCPR Classroom group showed significantly more adequate depth than the control group $(p=0.001)$. There were $39.0 \%$ (95\% Cl 33.8 to 44.2 ; $\mathrm{p}<0.0001)$ and $20.0 \%$ improvements $(95 \% \mathrm{Cl} 15.4$ to 24.7 ; $\mathrm{P}<0.0001)$ in the QCPR Classroom and control groups, respectively. The difference in adequate recoil between pretraining and post-training was $2.7 \%(95 \% \mathrm{Cl}-1.7$ to
Strengths and limitations of this study

- One of the advantages of Cardiopulmonary Resuscitation (CPR) training using the Quality Cardiopulmonary Resuscitation (QCPR) Classroom concept is good educational achievement with fewer instructors.

- Arranging objective real-time feedback on a big screen in front of everyone, visible to both instructor and students, significantly improved CPR quality.

- The QCPR Classroom group had $13.6 \%$ better adequate depth and $20.5 \%$ better adequate recoil than the control group.

- No retention measurement was taken, and the measurements were of all students together.

- This was manikin-based training, so it was not the same as in real life since the chest was not as hard as the human body.

7.1 ; pre $64.2 \pm 36.5 \%$ vs post $66.9 \% \pm 34.6 \%$; $p=0.23$ ) and $22.6 \%$ in the control and QCPR Classroom groups $(95 \% \mathrm{Cl}$ 17.8 to 27.3 ; pre $64.8 \pm 37.5 \%$ vs post $87.4 \% \pm 22.9 \%$; $\mathrm{p}<0.0001$ ), respectively.

Conclusions QCPR Classroom helped students achieve high-quality CPR training, especially for proper compression depth and full recoil. For good educational achievement, a novel QCPR Classroom with a metronome sound is recommended.

\section{INTRODUCTION}

The burden of cardiovascular diseases and the increasing number of out-of-hospital cardiac arrest (OHCA) cases remain a global concern. ${ }^{1}$ Performing bystander Cardiopulmonary Resuscitation (CPR) is one factor that can increase the survival rate of OHCA. ${ }^{2-7}$ 
The survival rate may be directly linked to the number of bystanders trained in CPR. ${ }^{2}$ As a complement to the 2015 American Heart Association (AHA) and Japan Resuscitation Council Guideline, the Global Resuscitation Alliance (GRA) was recently established to improve OHCA survival, and high-performance CPR was highlighted: a push depth of 5-6cm, rate of 100-120 compressions per minute (cpm), full recoil and minimising interruptions for chest compressions (less than $10 \mathrm{~s}$ ) ${ }^{8-10}$ In the GRA consensus, mandatory School and Community CPR was listed as one of the 10 steps to increase OHCA survival. ${ }^{8}$

Healthcare professionals were found to perform incomplete compression recoil in $46 \%$ of all cases and in $23.4 \%$ of cases involving paediatric patients. ${ }^{11}{ }^{12}$ Incomplete recoil leads to less blood flow to the brain, ${ }^{8}$ which causes coronary and cerebral perfusion pressure to deteriorate. ${ }^{13}$ Full recoil is one of the most important concepts for ensuring high-quality CPR. ${ }^{1014} 15$ Teaching individuals to compress the chest $5 \mathrm{~cm}$ deep and allow for full chest recoil is difficult during training without a feedback device.

We are currently facing a period of transition to CPR training with a feedback device as various feedback devices have been introduced, and research has supported their effectiveness. ${ }^{16-28}$ However, they are unavailable for CPR training targeting a large population. Laerdal Medical (Stavanger, Norway) launched the 'Quality Cardiopulmonary Resuscitation (QCPR) Classroom' concept, which provides real-time visual feedback for a greater number of participants at once. The effectiveness of the QCPR Classroom device was recently demonstrated by Kong et al. ${ }^{29}$ This study aims to examine the effectiveness of CPR skills delivered with QCPR Classroom feedback compared with standard CPR training. The hypothesis was that QCPR Classroom would generate higher achievement in CPR skill regardless of instructors' teaching skill. We aimed to determine whether QCPR Classroom could be the best practical model for CPR training.

\section{METHODS}

\section{Patient and public involvement}

Patients and/or the public were not involved in this study. The study population was focused on CPR trainees.

\section{Study population and design}

Prior to CPR training and study enrolment, oral informed consent was obtained from all participants. The study was approved by the Institutional Review Board at Kokushikan University. Sample size calculation was based on adequate depth from our pilot trial in which we examined the effect of the QCPR Classroom. We calculated a sample size of 232 with $90 \%$ power to detect a percentage of adequate compression depth between two groups $(\alpha=0.05)$. To reach a power of $90 \%, 41$ participants were needed per group in parallel, and the design effect was $5.66 ; 15 \%$ was detected as a deficit due to a mechanical issue, so 272 per group was calculated as a sample size. A total of 642 people were recruited from among CPR trainees enrolled in the Heart Saver Japan CPR training, which was held between March and September 2017. The inclusion criterion was age over 15 years. The exclusion criteria were the presence of upper extremity injury within the past 6 months and working as a healthcare professional regularly involved in resuscitation, such as Emergency Medical Technicians, paramedics and emergency room physicians or nurses. Previous CPR training status and quantity and timing of previous trainings were not used as inclusion or exclusion criteria.

\section{Measurements}

The primary outcome was compression depth $(\mathrm{mm})$, compression rate (cpm), adequate depth (\%) and adequate recoil (\%). These measurements were obtained both pretraining and immediately post-training. No feedback was provided to the participants. The measurement duration was $1 \mathrm{~min}$ as the bystander should change every minute during chest compression-only CPR. ${ }^{30}$ This was because the quality of chest compression-only CPR was demonstrated to decrease due to fatigue, and this can alter the results. The scores from a survey conducted after the training were considered a secondary outcome. The survey included the participants' confidence about the rate, depth and recoil pretraining and post-training as well as the ease of understanding feedback from the instructor. The survey is available in the online supplementary appendix 1.

\section{Study procedure}

A cluster randomised controlled trial was used (allocation ratio 1:1). A researcher (RS) generated a randomization list, and block-two randomization was performed. RS was not involved in the data collection and worked on statistics independently. Each session was randomly assigned to the intervention (standard or QCPR Classroom). Four lead instructors who had worked as healthcare professionals and had over 5 years of experience teaching CPR were selected from the Heart Saver Japan organisation. Data were collected during the Heart Saver Japan CPR+Automated External Defibrillator (AED) training sessions. The training focused on Basic Life Support, including CPR skills and AED, according to the Japan Resuscitation Council 2015 Guideline. Students were not medical personnel, so we instructed compression-only CPR. No preassignment or e-learning was given. The training started with a PowerPoint presentation-based instructor-led lecture followed by psychomotor practice. Psychomotor practice focused on chest-compression CPR. The sequence of the psychomotor practice is as follows: (1) Keys of compression: depth, rate, recoil; (2) single-rescuer chest compression-only CPR; (3) two-bystander chest compression-only CPR focusing on minimising interruptions; (4) check respiration; (5) scene safety, check consciousness and call for help; (6) use of AED; (7) practice from 'scene safety' to 'resume chest compression after giving a shock'; and (8) scenario-based 


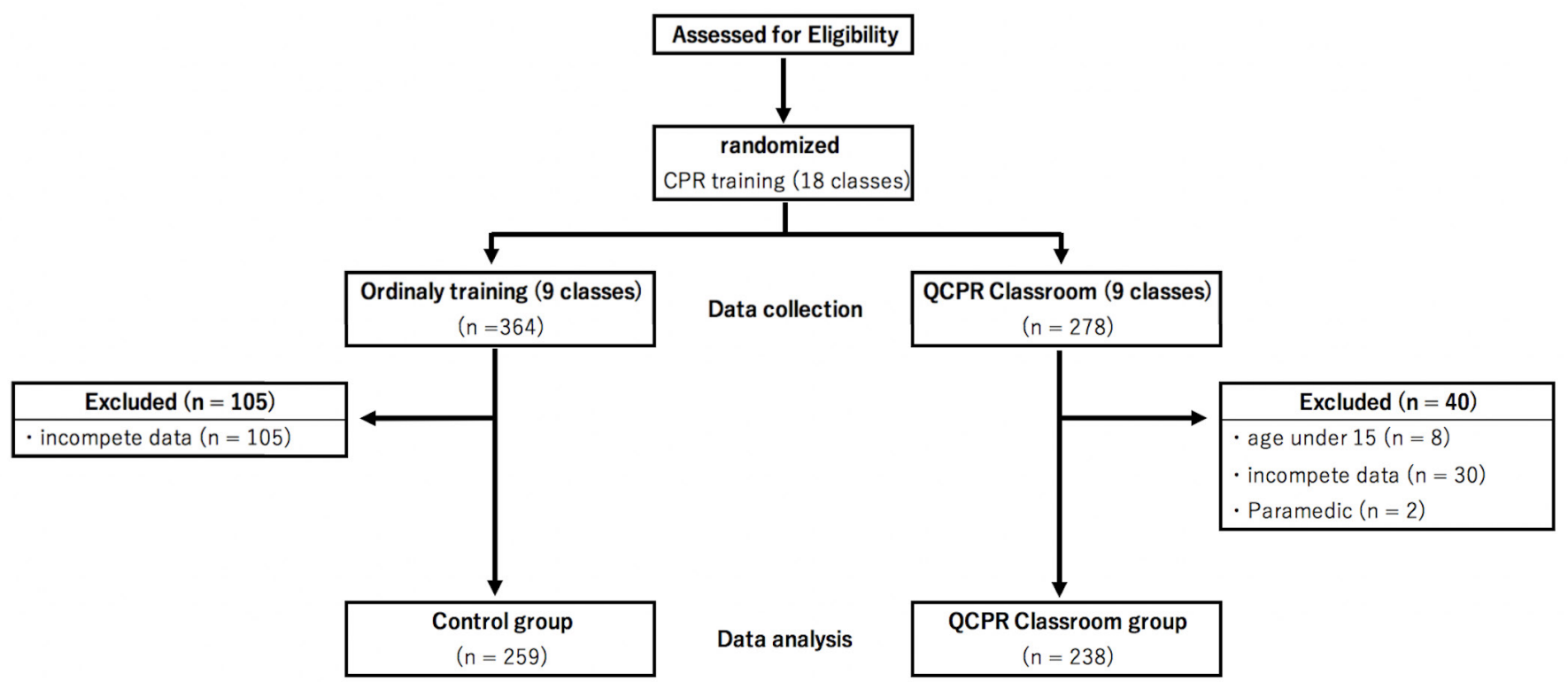

Figure 1 Flow chart of the study. CPR, Cardiopulmonary Resuscitation; QCPR, Quality Cardiopulmonary Resuscitation.

training. A total of $18 \mathrm{CPR}$ training sessions were studied with nine standard CPR training (control group) and nine QCPR Classroom sessions (figure 1).

In the QCPR Classroom group, participants received subjective and objective feedback from the instructor based on real-time feedback through the manikin, and participants were able to correct themselves based on feedback displayed on the screen of the device (figure 2). The control group received instructor's subjective feedback, so a hand clap was used based on the instructor's experience instead of using a metronome sound. As the AHA stated, audio-visual feedback is mandated for use. ${ }^{31}$ QCPR Classroom only provides visual feedback, so
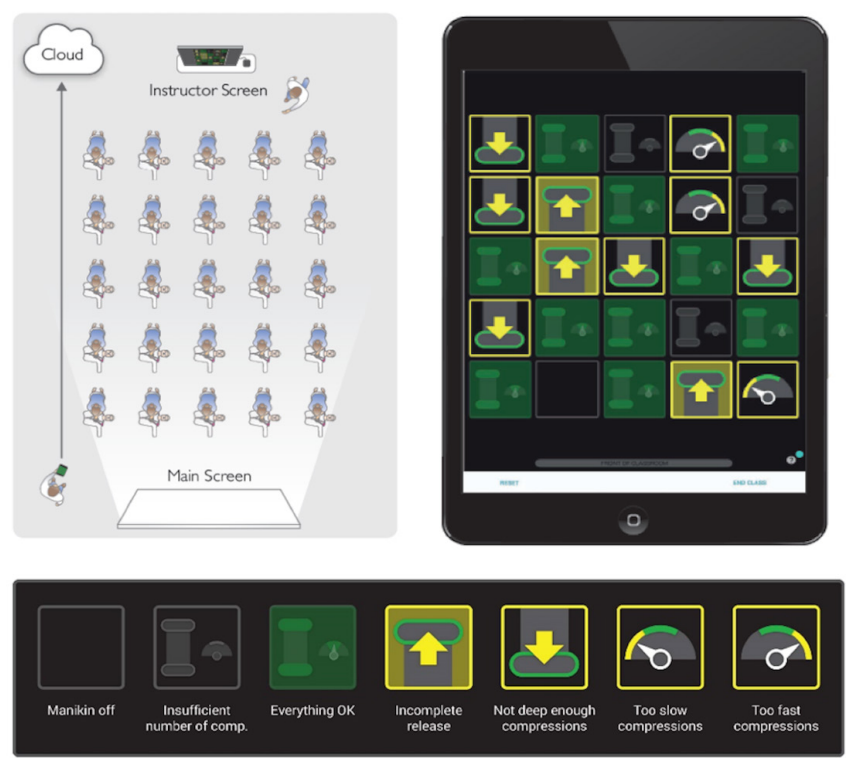

Figure 2 Image of Quality Cardiopulmonary ResuscitationClassroom feedback system. we used sound as an auditory aid for instruction in the QCPR Classroom group.

To measure the effect of CPR training, 1 min of chest compression was measured without any feedback given as a pretest. Similarly, $1 \mathrm{~min}$ of chest compression was also measured after the training as a post-test. Although $1 \mathrm{~min}$ of measurement may not be sufficient for CPR performance in real life, we focused on the initial CPR performance in a single-rescuer situation. A survey and baseline characteristics, such as weight, height and CPR training experience (table 1), were also collected after the posttraining measurement. The metronome was set at 110 beats/min and used for every instance of hands-on practice during the QCPR Classroom session, but no metronome was used during the standard CPR training.

Table 1 Demographic characteristics

\begin{tabular}{|c|c|c|c|}
\hline & $\begin{array}{l}\text { Control } \\
(n=259)\end{array}$ & $\begin{array}{l}\text { QCPR } \\
\text { Classroom } \\
(n=238)\end{array}$ & $P$ value \\
\hline Age, mean $\pm S D$ & $22.4 \pm 9.0$ & $19.4 \pm 5.6$ & $<0.0001^{*}$ \\
\hline Age, median (IQR) & 19 (17-23.5) & $17(16-21)$ & $<0.0001^{\star}$ \\
\hline Male, (\%) & $130(50.2)$ & $101(42.4)$ & 0.08 \\
\hline Height, mean \pm SD & $164.5 \pm 14.3$ & $164.2 \pm 8.2$ & 0.47 \\
\hline Weight, mean \pm SD & $57.9 \pm 12.1$ & $56.0 \pm 9.6$ & 0.06 \\
\hline $\mathrm{BMI}, \mathrm{mean} \pm \mathrm{SD}$ & $21.1 \pm 3.1$ & $20.7 \pm 2.6$ & 0.07 \\
\hline CPR training, $(\%)$ & $203(78.4)$ & $170(71.4)$ & 0.07 \\
\hline $\begin{array}{l}\text { CPR training within } \\
1 \text { year, }(\%)\end{array}$ & $89(41.2)$ & $63(36.4)$ & 0.34 \\
\hline
\end{tabular}

${ }^{*} \mathrm{p}<0.05$ significant.

BMI, body mass index; CPR, Cardiopulmonary Resuscitation; QCPR, Quality Cardiopulmonary Resuscitation; IQR, interquartile range 


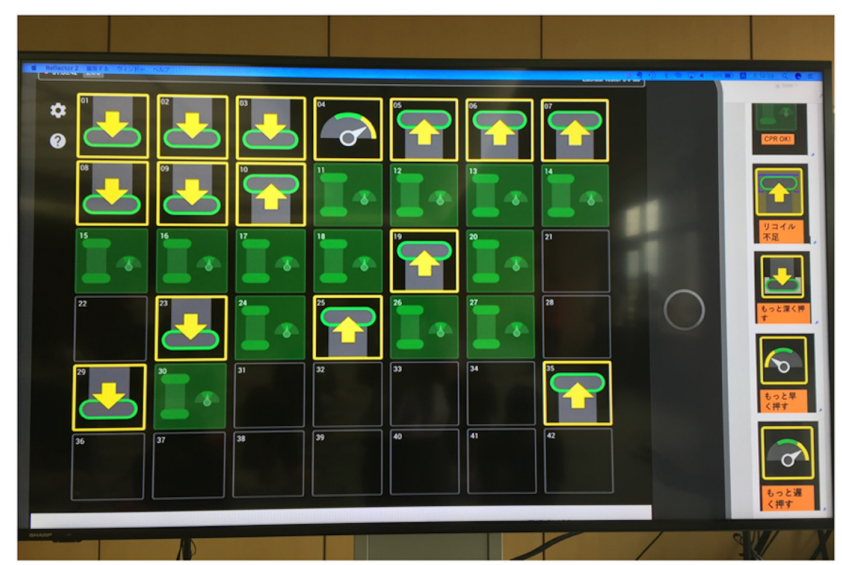

Figure 3 Image of actual display on the front screen.

\section{Instrumentation}

Compression data were registered using the Laerdal QCPR Classroom manikin system (Laerdal Medical, Stavanger, Norway), as shown in figure 2. This prototype system for community CPR training provides real-time visual feedback from 42 manikins while icons representing CPR performance from each manikin are visualised on an iPad tablet. We mirrored the iPad screen on a laptop using the application Reflector 2 (Squirrels, North Canton, Ohio, USA) to present real-time feedback on a large screen at the front of the classroom (figure 3). QCPR Classroom uses Laerdal Little Anne manikins, and each is instrumented with an optical compression sensor and microcontroller. The microcontroller analyses the signal from the compression sensor and calculates the number, depth and rate of compressions and incomplete release. A compression score is calculated using the rate, depth and release. Each sensor was checked for depth accuracy using a calibrated compression machine with $\pm 15 \%$ considered to indicate acceptable error.

The microcontroller also compares the compression performance with guidelines from the 2015 AHA requirements. Deviations from the guidelines are reported as 'too shallow,' 'incomplete release,' 'too fast' or 'too slow,' and deviation in each factor is presented as yellow icons on the tablet. If the compression performance is good, a green 'Everything OK' icon is presented. Data from the tablet is sent to a Microsoft Azure cloud service and made available as downloadable .csv files, which include the following parameters from each manikin and CPR session: the number of compressions, average compression rate, average compression depth, number of compressions with adequate depth, number of compressions with acceptable release, compression score, time and location of use. The control group also used the same Laerdal Little Anne manikins. However, we did not use a screen to show students objective feedback. During the training in the control group, lead-instructors were not allowed to access iPad, so they only gave subjective feedback to the students.

\section{Statistical analysis}

The rate and depth measurements are shown as the mean and SD. Normal distributions and homogeneity of variance were confirmed by a Q-Q plot. The differences and $95 \%$ confidence intervals are shown in tables. The adequate depth and recoil were calculated as percentages. The difference between pretraining and post-training measurements within the groups were analysed using a paired t-test and McNemar test. Group comparison for both pretraining and post-training was conducted using Welch's t-test and $\chi^{2}$ test. The medians and interquartile ranges (IQRs) are presented for ordinal data. We compared the groups using the Wilcoxon single-rank test. The data were analysed using JMP V.11.2.0 (SAS Institute), and $\mathrm{p}$ values less than 0.05 were considered significant.

\section{RESULTS}

\section{Demographic characteristics}

A total of 642 people participated in this study. As shown in figure 1, 145 participants were excluded due to incomplete data $(n=135)$, age under 15 years $(n=8)$ and being paramedics $(n=2)$. Significant age difference between the groups was found $(p \leq 0.0001)$. A statistical difference was found in age among individuals in clusters, but the $\mathrm{SD}$ and IQR overlapped. The age variation was not clinically significant due to overlap of precision measured. After the CPR training, 497 participants were eligible for analysis with 259 in the control group and 238 in the QCPR Classroom group. The demographic characteristics are shown in table 1.

\section{Primary outcome}

All chest-compression parameters at pretraining and posttraining are shown in table 2. After the training, the mean compression depth of each student was $56.1 \pm 9.8 \mathrm{~mm}$ in the control group and $59.5 \pm 7.9 \mathrm{~mm}$ in the QCPR Classroom group. Significantly more participants in the QCPR Classroom group achieved higher adequate depth compared with the control group $(\mathrm{p}<0.001$; table 3$)$. In the QCPR Classroom group, there was an improvement of $39.0 \%$ (95\% CI 33.8 to 44.2 ) in the percentage of adequate depth. In the control group, the improvement was $20.0 \%$ (95\% CI 15.4 to 24.7; $\mathrm{p}<0.0001$; table 2).

Both groups demonstrated average compression rates of 100-120 cpm (table 2). A statistically significant difference was found between groups in terms of recoil $(\mathrm{p}<0.001 ;$ table 3$)$. The control group demonstrated a $2.7 \%$ (95\% CI -1.7 to 7.1$)$ increase in the percentage of recoil ( $\mathrm{p}=0.23$ ). The QCPR Classroom group demonstrated a $22.6 \%$ (95\% CI 17.8 to 27.3$)$ increase in the percentage of recoil $(\mathrm{p}<0.0001$; table 2$)$.

\section{Secondary outcome}

The survey included participants' confidence levels before and after training regarding three parameters (rate, depth and recoil) using the following question: ' $O n$ 


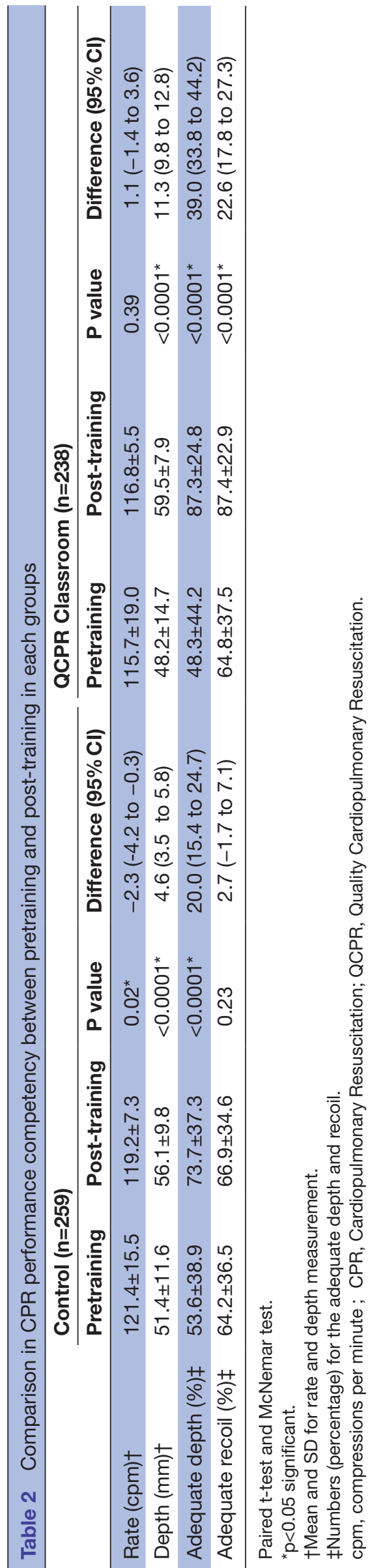

a scale of 1-10, with 1 being not confident and 10 being very confident, how much confidence do you have to perform chest compressions?' Confidence regarding CPR performance did not differ between groups. The question 'How do you rate the ease of understanding the feedback from the instructor?' was asked to address the ease of understanding. A significance difference was seen regarding the rate of feedback from the instructor; the QCPR Classroom training group (10.0 (9.0-10.0)) showed higher scores than the control group (10.0 (8.5-10.0]; $\mathrm{p}=0.01$; table 4$)$.

\section{DISCUSSION}

The 2015 AHA Guidelines recommend and the AHA mandated in 2017 use of audiovisual feedback in all CPR training. ${ }^{81431}$ Previous studies indicate that use of feedback for CPR performance significantly improves CPR quality. ${ }^{16-28}$ In our study, CPR quality was significantly better in the QCPR Classroom than in the control group. The compression depth was $59.5 \pm 7.9 \mathrm{~mm}$ in the QCPR Classroom group and $56.1 \pm 9.8 \mathrm{~mm}$ in the control group post-training. One hundred five and 90 participants in the QCPR Classroom and control groups, respectively, compressed greater than $60 \mathrm{~mm}$. None in the QCPR Classroom group performed below $50 \mathrm{~mm}$ in the post-test, but 64 in the control group still did not reach $50 \mathrm{~mm}$.

Skorning et al found that correct depth was achieved in $73.1 \%$ of participants who used a feedback device and $45.2 \%$ of those who did not. ${ }^{20}$ Cortegiani et al tested a QCPR-Skillreporter feedback model where school children were allowed to practice 2 min compression-only CPR sessions while receiving real-time feedback on a computer screen, aiming for a $60 \%$ overall compression score. ${ }^{32}$ Following practice, the students received overall performance feedback from the computer. This model used equipment designed for smaller groups. While QCPR Classroom can handle 42 manikins simultaneously and feedback is given on only one parameter at the time, Cortegiani et al used a system that can handle six manikins simultaneously, giving feedback on depth, rate and leaning simultaneously. ${ }^{32}$

Various types of manikins have been used for CPR training, but the quality of CPR training depends on the instructor, and little is known about training quality. The GRA has highlighted the importance of high-performance CPR, and knowledge is needed in the general population. Significant age difference was found, but previous studies indicated that chest compression delivered by children aged 13-14years was similar to adult performance, and the researchers concluded that the performance depended on weight, age, ${ }^{33}$ height, BMI (body mass index) and sex. ${ }^{34}$ In our study, the median and IQR for each group were 19 (17-23) and 17 (16-21), there was no height or weight difference between groups, and both groups included participants over 15 years old, so we considered that the age difference was clinically negligible for adequate CPR performance. 
Table 3 The difference of CPR performance competency between the control group and QCPR-Classroom group at pretraining and post-training

\begin{tabular}{|c|c|c|c|c|}
\hline & Control $(n=259)$ & $\begin{array}{l}\text { QCPR Classroom } \\
(n=238)\end{array}$ & $P$ value & Difference $(95 \% \mathrm{Cl})$ \\
\hline \multicolumn{5}{|l|}{ Pretraining test } \\
\hline Rate (cpm)† & $121.4 \pm 15.5$ & $115.7 \pm 19.0$ & $<0.001^{*}$ & $-5.7(-8.7$ to -2.6$)$ \\
\hline Depth $(\mathrm{mm}) \dagger$ & $51.4 \pm 11.6$ & $48.2 \pm 14.7$ & $0.008^{\star}$ & $-3.2(-5.5$ to -0.85$)$ \\
\hline Adequate depth (\%)‡ & $53.6 \pm 38.9$ & $48.3 \pm 44.2$ & 0.15 & $-5.3(--12.7$ to 2.0$)$ \\
\hline Adequate recoil (\%)‡ & $64.2 \pm 36.5$ & $64.8 \pm 37.5$ & 0.84 & $3.3(-5.9$ to 7.2$)$ \\
\hline \multicolumn{5}{|l|}{ Post-training test } \\
\hline Rate $(\mathrm{cpm}) \dagger$ & $119.2 \pm 7.3$ & $116.8 \pm 5.5$ & $<0.001^{*}$ & $-2.3(-3.5$ to -1.2$)$ \\
\hline Depth $(\mathrm{mm}) \dagger$ & $56.1 \pm 9.8$ & $59.5 \pm 7.9$ & $<0.001^{*}$ & $3.5(1.9$ to 5.1$)$ \\
\hline Adequate depth (\%)‡ & $73.7 \pm 37.3$ & $87.3 \pm 24.8$ & $<0.001^{\star}$ & 13.6 (8.0 to 19.2$)$ \\
\hline Adequate recoil (\%)‡ & $66.9 \pm 34.6$ & $87.4 \pm 22.9$ & $<0.001^{*}$ & 20.5 (15.3 to 25.7 ) \\
\hline
\end{tabular}

Welch's t-test and $\mathrm{X}^{2}$ test.

${ }^{*} \mathrm{p}<0.05$ significant.

†Mean and SD for rate and depth measurement.

$\ddagger$ Numbers (percentage) for the adequate depth and recoil.

cpm, compressions per minute; CPR: Cardiopulmonary Resuscitation; QCPR, Quality Cardiopulmonary Resuscitation.

Healthcare professionals were previously found to perform incomplete chest recoil in $46 \%$ of cases. ${ }^{11}$ Teaching the concept of recoil is not easy; we found only a $2.7 \%$ increase in the control group. Contri et al stated that instruction about recoil must be modified according to the participants' physical characteristics. ${ }^{34}$ However, during standard community CPR training, the instructor cannot spend much time on each individual to correct their performance.
This novel 'QCPR Classroom' is a unique real-time visual in-action feedback system that has significantly impacted CPR performance with 42 manikins providing feedback simultaneously in a large group-training setting. As Kong et al concluded, overall CPR quality was improved through QCPR Classroom-based training. ${ }^{29}$ The QCPR Classroom group showed significant improvement in CPR skills between post-training and pretraining, especially in the percentage of adequate depth and recoil. The purpose

Table 4 Survey regarding the ease of understanding the feedback from instructor and confidence levels before and after training on three parameters (rate, depth and recoil)
Question
Control $(n=259)$
QCPR Classroom ( $n=238)$
P value

How much confidence do you have to perform chest compressions before training?*

$\begin{array}{llll}\text { Rate } & 5.0(3.0-8.0) & 5.0(3.0-7.0) & 0.33 \\ \text { Depth } & 5.0(3.0-7.5) & 5.0(3.0-7.0) & 0.27 \\ \text { Recoil } & 5.0(3.0-7.0) & 5.0(3.0-7.0) & 0.37\end{array}$

How much confidence do you have to perform chest compressions after training? ${ }^{\star}$

$\begin{array}{llll}\text { Rate } & 8.0(7.0-9.0) & 8.0(7.0-9.0) & 0.98 \\ \text { Depth } & 8.0(7.0-9.0) & 8.0(7.0-9.0) & 0.96 \\ \text { Recoil } & 8.0(7.0-9.0) & 8.0(7.0-9.0) & 0.76\end{array}$

How do you rate the ease of understanding the feedback from instructor? $\dagger$

\begin{tabular}{llll} 
Rate & $10.0(8.5-10.0)$ & $10.0(9.0-10.0)$ & $0.01^{*}$ \\
Depth & $10.0(8.0-10.0)$ & $10.0(9.0-10.0)$ & 0.08 \\
Recoil & $10.0(8.0-10.0)$ & $10.0(9.0-10.0)$ & 0.12 \\
\hline
\end{tabular}

Wilcoxon test, Median (IQR).

*The survey was rated on 'On a scale 1 to 10, with 1 being not confident and 10 being very confident, how much confidence do you have to perform chest compression before and after training?'

†The survey was rated on 'On a scale 1 to 10, with 1 being very difficult and 10 being very easy, how do you rate the ease of understanding the feedback from instructor?'

QCPR, Quality Cardiopulmonary Resuscitation. 
of QCPR Classroom is to make it easy to objectively measure and improve CPR performance in community CPR classes. In a large classroom, learners could see their CPR performance on a big screen at the front of the class. Kong et al randomised the groups, and only the instructor saw the feedback icon on the iPad; they examined how 'QCPR Classroom' training affects CPR performance improvement of laypeople in the large group setting. ${ }^{29}$ Students only received objective feedback directly from the instructor. Our study randomised the groups, and both students and instructor were able to see the feedback icon on the iPad by arranging them on a big screen at the front of the classroom, allowing students to have real-time feedback. It is possible to provide high-quality CPR training with a lead-instructor:manikin:students ratio of 1:42:84, enabling good educational achievement with fewer instructors.

CPR training has been studied for decades by observing participants and comparing their performance to guidelines. The findings show that training does not provide sufficient practice, ${ }^{35} 36$ it does not include Dispatcher-Assisted $\mathrm{CPR},{ }^{37}$ participants lack preparedness for real situations, ${ }^{36} 3839$ and objective student feedback and assessment are not performed. ${ }^{40}$ In 1991, Kaye et al reported that instructors made CPR courses by themselves and included only $10 \mathrm{~min}$ of practical training. ${ }^{35}$ The instructors also performed subjective assessments of the students to let the students pass the course even though the students would not have passed according to objective measurements or evaluation by researchers. ${ }^{35}$

The need for standardised training, more relevant training, and objective assessment has been known since the early 1990s, but most training teaches laypeople to perform CPR alone without dispatcher assistance, and they practice CPR without feedback or performance assessment. We generally do not know what quality of CPR participants will perform during resuscitation, but we know that good-quality bystander CPR is positively reflected in survival. ${ }^{41-44}$ It is possible to make training for laypeople more relevant and effective by focusing on the most important learning objectives, prioritising practical training, training people to work in teams with dispatchers, using objective feedback to stimulate good performance, and documenting the results for quality improvement and cultivating a culture of excellence. QCPR Classroom can provide objective feedback on the quality and quantity of CPR.

The use of feedback in a hospital setting ${ }^{16}$ as well as in the Emergency Medical Services field was suggested. ${ }^{17}$ Tanaka $e t$ al also suggested the implementation of feedback devices in athletic training. ${ }^{45}$ The use of such a device is also highly recommended even for healthcare professionals. Laypeople who may encounter situations of cardiac arrest rarely need to use a feedback device to deliver high-quality CPR, which may be directly linked to the chances of survival. An AED with a feedback device is the best method for citizens to deliver higher-quality CPR. We believe that the combination of training with QCPR
Classroom and performing CPR with a feedback device in the field would have a positive impact on survival rates.

Recoil is the most difficult part for participants to perform within such a short time, especially for those training in CPR for the first time. The confidence level of learners toward recoil was 8.0/10.0 in our study. The hands are off the sternum when teaching full recoil, and incomplete release would occur if the recoil concept was not mentioned. In our opinion, instructors prioritise teaching the concepts of depth and rate rather than recoil because feedback on recoil cannot be given as subjectively. In standard CPR training, we assume that the main focus of participants tends to be compressing harder; therefore, participants easily forget recoil and neglect to perform it. QCPR Classroom significantly improved recoil performance, although it did not influence the confidence level. With achieving appropriate depth, rate and recoil, performing good chest compression leads to favourable outcomes. Recent guidelines increasingly emphasise the necessity of high-quality CPR performance by not only Emergency Medical Technicians or first responders but also citizens. ${ }^{46}$

With the highlighted importance of objective feedback during CPR training, we hope this pilot study on QCPR Classroom training could be considered as a model for future CPR training. The role of instructors is to emphasise the importance of bystander CPR and Public Access Defibrillation. Therefore, instead of focusing too much on the recoil or another part of high-quality CPR, the importance of immediate initiation of CPR without hesitating should be highlighted during the training. It is still very important to determine how to design these environments and prioritise emergency action plans, such as contacting Emergency Medical Services personnel and summoning other people.

\section{Study limitations}

A strength of this study was that arranging objective realtime feedback on a big screen in front of everyone, visible to both instructor and students, significantly improved CPR quality. Our study has several limitations. First, the manikins' chest is not as hard as the human body, so chest compressions are not the same as in real life. Second, this study was conducted using CPR training that targeted a large amount of laypeople, who all performed CPR together. Since chest compression was tested in this environment, the rate measurement may have been influenced by other participants. The metronome was used in the QCPR Classroom group only; no metronome was used in the control group. Third, the instructors' knowledge may have been questionable since the instructors were learning about the device as the training proceeded. Fourth, the survey was collected postcourse including questions about the participants' confidence prior to the training, which may add a large element of recall bias. Fifth, there is a potential lack of generalizability since the quality of instructors and the standards of training may vary in other settings. Sixth, participants were lost due to 
lack of data. This occurred because of a mechanical issue, and CPR skill data were not well registered on the cloud. Finally, the study only measured short-term improvement, not retention.

\section{CONCLUSION}

The use of a novel 'QCPR Classroom' prototype to educate a large group of laypeople with real-time visual CPR feedback has been described, and the effectiveness of training was assessed. The QCPR Classroom training achieved a higher percentage of adequate depth and recoil than the standard training with subjective assessment by instructors and a higher percentage of adequate recoil. During in-action 'QCPR Classroom' training with a metronome sound, displaying all students' feedback on the big screen significantly provided accurate real-time visual feedback to achieve two important components together: compressing the chest with a depth over $5 \mathrm{~cm}$ and minimising the incomplete release of the chest. Teaching CPR to larger groups of laypeople with a realtime feedback system, a novel QCPR Classroom with a metronome sound, is a recommended CPR training model.

\section{Author affiliations}

${ }^{1}$ Research Institute of Disaster Management and EMS, Kokushikan University, Tama City, Japan

${ }^{2}$ Graduate School of EMS System, Kokushikan University, Tama City, Japan ${ }^{3}$ Laerdal Medical Cooperation, Stavanger, Norway

${ }^{4}$ Faculty of Emergency Medical Science, Meiji University of Integrative Medicine, Kyoto, Japan

Acknowledgements The authors wish to thank all staff members at the Research Institute of Disaster Management and EMS at Kokushikan University for their assistance in this study. The authors also thank Laerdal Medical for their assistance in using QCPR Classroom.

Contributors ST carried out all the studies, participated in the sequence alignment and drafted the manuscript. ST, KT, TH, RS, HM, TSB, HTaky and HTana were involved in the study design. ST, KT, TH and HTana contributed to the study implementation. HM and TSB developed the concept and contributed to technological support. ST, KT, TH, HTaka, Al, HU, YK, MY and HTana were involved in the data collection. RS conducted statistical analysis. ST, RS and HTana performed data analysis. HTaky, HM and TSB contributed critical revisions to the manuscript. HTana also revised and approved the final manuscript. All authors read and approved the final submission.

Funding The authors have not declared a specific grant for this research from any funding agency in the public, commercial or not-for-profit sectors.

Competing interests TSB and HM are employees of Laerdal Medical. They contributed the QCPR Classroom prototype, study design and critical revision of the manuscript, but had no role in data collection, analysis of the results, or decision to publish. Other authors did not receive any payment, gift or anything from Laerdal Medical.

Patient consent for publication Obtained.

Ethics approval The study was approved by the Institutional ReviewBoard at Kokushikan University, Japan under the registration number 16-RI002 on February 23rd, 2017.

Provenance and peer review Not commissioned; externally peer reviewed.

Data sharing statement All relevant data are within the paper and its Supporting Information files.

Open access This is an open access article distributed in accordance with the Creative Commons Attribution Non Commercial (CC BY-NC 4.0) license, which permits others to distribute, remix, adapt, build upon this work non-commercially, and license their derivative works on different terms, provided the original work is properly cited, appropriate credit is given, any changes made indicated, and the use is non-commercial. See: http://creativecommons.org/licenses/by-nc/4.0/.

\section{REFERENCES}

1. Mozaffarian D, Benjamin EJ, Go AS, As G, et al. Heart disease and stroke statistics-2016 update: a report from the American Heart Association. Circulation 2016;133:e38-e360.

2. Hasselqvist-Ax I, Riva G, Herlitz J, et al. Early cardiopulmonary resuscitation in out-of-hospital cardiac arrest. $N$ Engl J Med 2015;372:2307-15

3. Wissenberg M, Lippert FK, Folke F, et al. Association of national initiatives to improve cardiac arrest management with rates of bystander intervention and patient survival after out-of-hospital cardiac arrest. JAMA 2013;310:1377-84.

4. Malta Hansen C, Kragholm K, Pearson DA, et al. Association of bystander and first-responder intervention with survival after out-of-hospital cardiac arrest in North Carolina, 2010-2013. JAMA 2015;314:255-64.

5. Iwami T, Kawamura T, Hiraide A, et al. Effectiveness of bystanderinitiated cardiac-only resuscitation for patients with out-of-hospital cardiac arrest. Circulation 2007;116:2900-7.

6. Takahashi H, Sagisaka R, Natsume Y, et al. Does dispatcher-assisted CPR generate the same outcomes as spontaneously delivered bystander CPR in Japan? Am J Emerg Med 2018;36:384-91.

7. Nakahara S, Tomio J, Ichikawa M, et al. Association of bystander interventions with neurologically intact survival among patients with bystander-witnessed out-of-hospital cardiac arrest in Japan. JAMA 2015;314:247-54.

8. American Heart Association. Highlights of the 2015 American Heart Association guidelines update for CPR and ECC. $2015 \mathrm{https}: / /$ eccguidelines.heart.org/wp-content/uploads/2015/10/2015-AHAGuidelines-Highlights-English.pdf (Accessed 1 Sep 2017).

9. Japan Resuscitation Council. Chapter 1 BLS: Basic Life Support. https://www.japanresuscitationcouncil.org/wp-content/uploads/ 2016/04/1327fc7d4e9a5dcd73732eb04c159a7b.pdf (Accessed 3 Feb 2019).

10. Global Resuscitation Alliance. Improving Survival from Out-ofHospital Cardiac Arrest: A Call to Establish a Global Resuscitation Alliance. http://www.laerdalevents.com/gra/wp-content/pdf/call_to_ establish.pdf (Accessed 2 Dec 2017).

11. Aufderheide TP, Pirrallo RG, Yannopoulos D, et al. Incomplete chest wall decompression: a clinical evaluation of CPR performance by EMS personnel and assessment of alternative manual chest compression-decompression techniques. Resuscitation 2005;64:353-62.

12. Sutton RM, Niles D, Nysaether J, et al. Quantitative analysis of CPR quality during in-hospital resuscitation of older children and adolescents. Pediatrics 2009;124:494-9.

13. Yannopoulos D, McKnite S, Aufderheide TP, et al. Effects of incomplete chest wall decompression during cardiopulmonary resuscitation on coronary and cerebral perfusion pressures in a porcine model of cardiac arrest. Resuscitation 2005;64:363-72.

14. Neumar RW, Shuster M, Callaway CW, et al. American Heart Association Guidelines Update for Cardiopulmonary Resuscitation and Emergency Cardiovascular Care, Part 1: Executive Summary. Circulation 2015;2015:S315-67.

15. Perkins GD, Handley AJ, Koster RW, et al. European Resuscitation Council Guidelines for Resuscitation 2015: Section 2. Adult basic life support and automated external defibrillation. Resuscitation 2015;95:81-99.

16. Abella BS, Edelson DP, Kim S, et al. CPR quality improvement during in-hospital cardiac arrest using a real-time audiovisual feedback system. Resuscitation 2007;73:54-61.

17. Hostler D, Everson-Stewart S, Rea TD, et al. Effect of real-time feedback during cardiopulmonary resuscitation outside hospital: prospective, cluster-randomised trial. BMJ 2011;342(d512):1-10.

18. Buléon C, Delaunay J, Parienti JJ, et al. Impact of a feedback device on chest compression quality during extended manikin CPR: a randomized crossover study. Am J Emerg Med 2016;34:1754-60.

19. Bobrow BJ, Vadeboncoeur TF, Stolz U, et al. The influence of scenario-based training and real-time audiovisual feedback on outof-hospital cardiopulmonary resuscitation quality and survival from out-of-hospital cardiac arrest. Ann Emerg Med 2013;62:47-56.

20. Skorning M, Beckers SK, Brokmann JCh, et al. New visual feedback device improves performance of chest compressions 
by professionals in simulated cardiac arrest. Resuscitation 2010;81:53-8.

21. Buléon C, Parienti JJ, Halbout L, et al. Improvement in chest compression quality using a feedback device (CPRmeter): a simulation randomized crossover study. Am J Emerg Med 2013;31:1457-61.

22. Kramer-Johansen J, Myklebust H, Wik L, et al. Quality of out-ofhospital cardiopulmonary resuscitation with real time automated feedback: a prospective interventional study. Resuscitation 2006;71:283-92.

23. Cheng A, Brown LL, Duff JP, et al. International Network for Simulation-Based Pediatric Innovation, Research, \& Education (INSPIRE) CPR Investigators. Improving cardiopulmonary resuscitation with a CPR feedback device and refresher simulations (CPR CARES Study): a randomized clinical trial. JAMA Pediatr 2015;169:137-44.

24. Cheng A, Overly F, Kessler D, et al. International Network for Simulation-Based Pediatric Innovation, Research, \& Education (INSPIRE) CPR Investigators. Perception of CPR quality: Influence of CPR feedback, Just-in-Time CPR training and provider role. Resuscitation 2015;87:44-50.

25. White $\mathrm{AE}, \mathrm{Ng} \mathrm{HX}, \mathrm{Ng} \mathrm{WY}$, et al. Measuring the effectiveness of a novel CPRcard feedback device during simulated chest compressions by non-healthcare workers. Singapore Med J 2017;58:438-45.

26. Tanaka S, White AE, Sagisaka R, et al. Comparison of quality of chest compressions during training of laypersons using Push Heart and Little Anne manikins using blinded CPRcards. Int Emerg Med 2017;10:20.

27. Baldi E, Cornara S, Contri E, et al. Real-time visual feedback during training improves laypersons' CPR quality: a randomized controlled manikin study. CJEM 2017;19:480-7.

28. Yeung J, Meeks R, Edelson D, et al. The use of CPR feedback/ prompt devices during training and CPR performance: A systematic review. Resuscitation 2009;80:743-51.

29. Kong SY, Shin SD, Song KJ, et al. Effect of instructor's realtime feedback using QCPR-classroom device during layperson cardiopulmonary resuscitation (CPR) training on quality of CPR Performances: A prospective cluster-randomised trial. BMJ Open 2018;8.

30. Nishiyama C, Iwami T, Kawamura T, et al. Quality of chest compressions during continuous CPR; comparison between chest compression-only CPR and conventional CPR. Resuscitation 2010;81:1152-5.

31. American Heart Association. AHA requirement on Use of feedback devices in adult CPR training courses. http://ahainstructornetwork. americanheart.org/idc/groups/ahaecc-public/@wcm/@ecc/ documents/downloadable/ucm_495639.pdf (Accessed 1 Sep 2017).
32. Cortegiani A, Russotto V, Montalto F, et al. Use of a Real-Time Training Software (Laerdal QCPR®) Compared to Instructor-Based Feedback for High-Quality Chest Compressions Acquisition in Secondary School Students: A Randomized Trial. PLoS One 2017:12:e0169591.

33. Jones I, Whitfield R, Colquhoun $\mathrm{M}$, et al. At what age can schoolchildren provide effective chest compressions? An observational study from the Heartstart UK schools training programme. BMJ 2007;334:1201.

34. Contri E, Cornara S, Somaschini A, et al. Complete chest recoil during laypersons' CPR: Is it a matter of weight? Am J Emerg Med 2017;35:1266-8.

35. Kaye W, Rallis SF, Mancini ME, et al. The problem of poor retention of cardiopulmonary resuscitation skills may lie with the instructor, not the learner or the curriculum. Resuscitation 1991;21:67-87.

36. Parnell MM, Larsen PD. Poor quality teaching in lay person CPR courses. Resuscitation 2007;73:271-8.

37. Wagner P, Lingemann C, Arntz HR, et al. Official lay basic life support courses in Germany: is delivered content up to date with the guidelines? An observational study. Emerg Med J 2015;32:547-52.

38. Møller TP, Hansen CM, Fjordholt M, et al. Debriefing bystanders of out-of-hospital cardiac arrest is valuable. Resuscitation 2014;85:1504-11.

39. Hirose $\mathrm{T}$, Iwami T, Ogura H, et al. Effectiveness of a simplified cardiopulmonary resuscitation training program for the non-medical staff of a university hospital. Scand J Trauma Resusc Emerg Med 2014;22:1-7.

40. Lynch B, Einspruch EL, Nichol G, et al. Assessment of BLS skills: optimizing use of instructor and manikin measures. Resuscitation 2008;76:233-43.

41. Wik L, Steen PA, Bircher NG. Quality of bystander cardiopulmonary resuscitation influences outcome after prehospital cardiac arrest. Resuscitation 1994;28:195-203.

42. Van Hoeyweghen RJ, Bossaert LL, Mullie A, et al. Quality and efficiency of bystander CPR. Resuscitation. Emerg Med J 2014:1-6.

43. Gallagher EJ, Lombardi G, Gennis P. Effectiveness of bystander cardiopulmonary resuscitation and survival following out-of-hospital cardiac arrest. JAMA 1995;274:1992-1925.

44. Takei $\mathrm{Y}$, Nishi $\mathrm{T}$, Matsubara $\mathrm{H}$, et al. Factors associated with quality of bystander CPR: the presence of multiple rescuers and bystanderinitiated CPR without instruction. Resuscitation 2014;85:492-8.

45. Tanaka S, Rodrigues W, Sotir S, et al. CPR performance in the presence of audiovisual feedback or football shoulder pads. BMJ Open Sport Exerc Med 2017;3:e000208.

46. Japan Resuscitation Council. Chapter 8 ETI: Education, Implementation, and Teams. http://www.japanresuscitationcouncil. org/wp-content/uploads/2016/04/b7b5b647189bc07f38f6fecf 014cf5d9.pdf (Accessed 2 Dec 2017). 